\title{
Recurrence risks for neural tube defects in siblings of patients with lipomyelomeningocele
}

Courtney Drake Sebold, $M S^{1}$, Elizabeth C. Melvin, MS, CGC ${ }^{1}$, Debbie Siegel ${ }^{1}$, Lorraine Mehltretter, MS, CGC ${ }^{1}$, David S. Enterline, $M D^{1}$, Jeffrey S. Nye, $M D, P h D^{2}$, John Kessler, $M D^{3}$, Alexander Bassuk, $M D, P h D^{3}$, Marcy C. Speer, $P h D^{1}$, Timothy M. George, $M D^{1}$, and the NTD Collaborative Group

\begin{abstract}
Purpose: Neural tube defects (NTDs) are a group of widely varying congenital malformations resulting from incomplete or improper fusion of the neural tube during embryonic development. NTDs are traditionally classified by the presence or absence of a layer of skin covering the spinal defect. Although a genetic component has been well established in the etiology of open NTDs, studies examining the genetics of closed NTDs such as lipomyelomeningocele are rare. We and others have previously observed families in which multiple members were affected with a broad spectrum of NTDs, suggesting the possibility of a common genetic etiology. Methods: We calculated the sibling recurrence risk in 52 pedigrees in which the proband was diagnosed with lipomyelomeningocele (LMM), defining recurrence broadly to include both closed and open neural tube defects. Results: Although no recurrences of LMM were observed among younger siblings, one younger sibling had myelomeningocele, yielding an estimate of recurrence risk of 0.04 (95\% $\mathrm{Cl}$ 0.01-0.20). When all siblings of the proband were included, two additional affected siblings were identified, one with anencephaly and another with fatty filum, yielding an estimate of recurrence risk of $0.043(95 \% \mathrm{Cl} 0.01-0.12)$. Conclusions: Although the sample size is small, these data are not inconsistent with recurrence risks for myelomeningocele, ranging from $2 \%$ to $5 \%$ in siblings. These data suggest the underlying genetic basis for closed defects may be the same or closely related to that for myelomeningocele in some families, although a larger sample will be necessary before these data are appropriate for use in a clinical setting. Further characterizations, including whether risk for recurrence of NTDs or LMM in families in which the proband is affected with LMM are altered by folate supplementation, may shed light on the underlying genetics.
\end{abstract}

Genet Med 2005:7(1):64-67.

Key Words: neural tube defects, closed NTDs, lipomyelomeningocele, congenital malformations, sibling recurrence

Neural tube defects (NTDs) are the second most common congenital malformation, occurring once in every 1000 live births. The term "NTD" encompasses a wide range of defects resulting from the abnormal fusion and development of the neural tube during embryonic development. NTDs can be classified according to a number of criteria, including vertebral level and degree of severity. Another commonly accepted method of classifying NTDs is the presence or absence of a layer of skin covering the spinal defect. Open NTDs, those without skin covering, occur when the brain and/or spinal cord is exposed at birth through a defect in the skull or vertebrae. The two most common forms of open NTDs are myelomeningocele and anencephaly.

From ${ }^{1}$ Duke University Medical Center, Durham, North Carolina; ${ }^{2}$ Johnson and Johnson PRD, Titusville, New Jersey; ${ }^{3}$ Northwestern University's Feinberg School of Medicine, Chicago, Illinois.

Marcy Speer, PhD, Center for Human Genetics, Duke University Medical Center, Box 3445, Durham, NC 27710.

Received: May 24, 2004.

Accepted: August 9, 2004.

DOI: 10.1097/01.GIM.0000151158.09278.2B
In contrast, closed NTDs, also known as occult spinal dysraphisms, have a layer of skin overlying the spinal defect. One common occult lesion is lipomyelomeningocele, characterized by fatty elements infiltrating through a vertebral defect into the spinal column. The prevalence of lipomyelomeningocele has been estimated to be 1 in 4000, with a female:male ratio of 1.5:1. ${ }^{1-3}$ However, this figure may underestimate the true prevalence because patients with lipomyelomeningocele are sometimes clinically asymptomatic. Alternatively, they may present with vague symptoms, such as leg pain and weakness or recurrent urinary tract infections and incontinence. ${ }^{3}$ Often, the only indication of a lipomyelomeningocele is a small dorsal cutaneous abnormality, such as a midline dimple, skin tag, or hemangioma. ${ }^{4}$ In the absence of an obvious dorsal fatty mass, some patients may be overlooked until more serious neurological problems arise. In addition, imaging modalities used before the development of the MRI were less sensitive and more likely to miss smaller defects. Thus, it has only been in recent years that this defect can be reliably diagnosed.

Open NTDs have a complex etiology, involving both genetic and environmental factors. A significant genetic component in the etiology of open NTDs is supported by several lines of 
evidence including associations with known genetic syndromes, ${ }^{5,6}$ associations with cytogenetic abnormalities, ${ }^{7,8}$ and a plethora of animal models. ${ }^{9,10}$ In addition, the recurrence risk in families with one member affected with an NTD $(2-5 \%)$ is 25- to 50-fold higher than the 1 in 1000 incidence of NTDs in the general population. ${ }^{11}$

Although the role of genetic factors in the etiology of open NTDs is unequivocal, it remains unclear whether the genetic basis for closed NTDs such as LMM is due to the same or different causes. Open and closed NTDs are thought to be due to different etiologies because they originate at different times during embryonic development. ${ }^{3}$ However, there is evidence that at least some of the underlying etiologic factors are common to both open and closed NTDs. We and others have previously reported families in which closed and open NTDs occur ${ }^{12-14}$; such families suggest an underlying gene(s) with pleiotropic effects. To investigate this hypothesis, we estimated the recurrence risk to siblings of a proband with lipomyelomeningocele and hypothesized that it would not be different than the recurrence risk to siblings of a proband with an open neural tube defect. We conclude that this similarity may suggest a common underlying gene(s) with pleiotropic effect.

\section{MATERIALS AND METHODS}

Families in this study included those in which the proband was diagnosed with lipomyelomeningocele. Ascertainment of these families occurred through a variety of resources, including web-based advertising, support groups such as the Lipomyelomeningocele Family Support Network (LFSN) and the Adult Tethered Cord Syndrome Support Group, e-mail listservs, and referrals from collaborating neurosurgeons. Detailed family history ${ }^{15}$ and pregnancy history information were collected. Diagnosis in affected family members was confirmed by review of imaging studies, operative reports, and/or medical records. Open neural tube defects included patients with anencephaly or myelomeningocele. Closed neural tube defects included lipomyelomeningocele and other spinal lipomas (myelocystoceles, terminal lipomas, fatty filum, intraspinal lipoma). Families in which nonproband members had lipomyelomeningocele were identified and tracked to document familial aggregation, but are not included in the recurrence risk calculations. This study was approved by the Duke University Medical Center Institutional Review Board and informed consent was obtained from all subjects.

\section{Recurrence risk}

Sibling recurrence risks were calculated using data collected from the standardized interviews. All siblings born subsequent to the proband were included in the calculation. The proportion of these siblings affected by a broad spectrum of neural tube defects, both open and closed, represents the sibling recurrence risk. Overall occurrence risk was calculated by including all siblings of the proband, regardless of the birth order. Confidence intervals (95\%) for the risk were calculated allowing for a continuity correction.

\section{RESULTS}

\section{Study population}

The sample used for this study consisted of 52 American Caucasian families in which the proband was diagnosed with lipomyelomeningocele. Of the 52 probands, 23 (44\%) were female and 29 (56\%) were male; this difference is not statistically significant. Of the cases, 15 (29\%) were confirmed by review of preoperative films; for the remaining 37 cases, preoperative films were not available and operative reports and/or medical records were evaluated.

\section{Sibling recurrence risk}

Recurrences are shown in Table 1. The sample population included a total of 52 families in which the proband was diagnosed with a lipomyelomeningocele. These families included 25 younger siblings; of these siblings, none were affected with lipomyelomeningocele. However, one sibling was diagnosed with myelomeningocele. The recurrence risk for this broad spectrum of neural tube defects is $4.0 \%$ (95\% CI 0.01-0.20). When all siblings of the proband with lipomyelomeningocele were considered, there were a total of 69 siblings, three of whom had NTD or related phenotypes (one myelomeningocele, one anencephaly, and one with fatty filum). Thus, the overall occurrence of a broad spectrum of neural tube defects in siblings of probands with lipomyelomeningocele is $4.3 \%$ (95\% CI 0.01-0.12).

\section{Familial aggregation}

Two families (8776 and 8237; Fig. 1) exhibited aggregation with affected individuals outside the nuclear family. In family 8776, a female with lipomyelomeningocele has a brother, a cousin, two second cousins, and 4th degree relative with lumbosacral myelomeningocele. In family 8237 , the proband with

Table 1

Phenotypes in siblings of 52 probands with lipomyelomeningocele

\begin{tabular}{lccccc}
\hline Diagnosis in: & Lipomyelomeningocele & Lumbosacral yelomeningocele & Anencephaly & Other spinal lipoma $^{a}$ & Unaffected \\
\hline Younger Siblings & 0 & 1 & 0 & 0 & 25 \\
All Siblings & 0 & 1 & 1 & $1^{b}$ & 69 \\
\hline
\end{tabular}

${ }^{a}$ OSL, other spinal lipomas (myelocystoceles, terminal lipomas, fatty filum, intraspinal lipomas).

${ }^{b}$ Older sibling had fatty filum. 


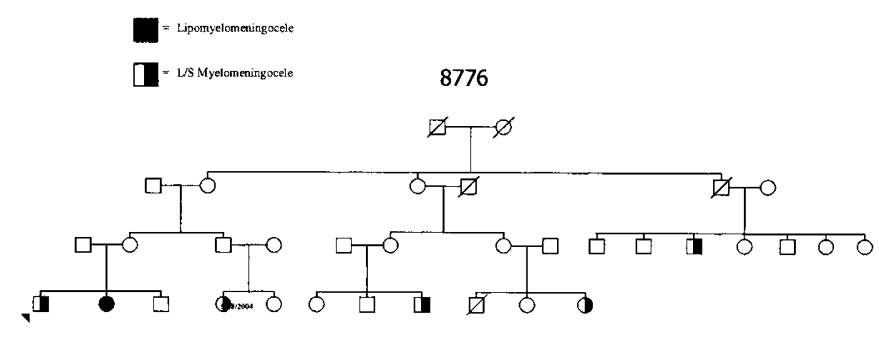

8237

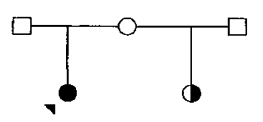

Fig. 1. Two families demonstrating occurrence of lipomyelomeningocele and other NTD outside of the nuclear family.

lipomyelomeningocele has a half-sibling with lumbosacral myelomeningocele.

\section{DISCUSSION}

The recurrence risk for a broad spectrum of neural tube defects in the siblings of probands affected with lipomyelomeningocele is consistent with the recurrence risk to siblings of probands with open neural tube defects, within the range of $2 \%$ to $5 \%$. These studies indicate open and closed NTDs may share the same or a similar underlying genetic mechanism, and they may represent the pleiotropic effects of a gene or genes segregating within these families. Although a large number of studies have examined the genetics of open NTDs, less attention has been focused on the genetics of closed NTDs. Carter et al. ${ }^{13}$ calculated the sibling recurrence risk for NTDs in 207 families in which the proband had a closed NTD, defined as any one of a spectrum of spinal cord abnormalities, including tethered cord, diastematomyelia, dermoid cysts, spina bifida occulta, and signs of neurological damage in conjunction with an identifiable spinal cord anomaly. In these families, the recurrence risk for any type of NTD was $4.12 \%$, similar to the established recurrence risk for open NTDs. However, given the wide phenotypic variation present within the sample, it is difficult to draw any conclusions about specific types of closed NTDs and their relationship to open NTDs. Furthermore, this study was done before the advent of magnetic resonance imaging studies, so its focus was on the most severe of cases as probands.

Other early studies examined the incidence of spina bifida occulta (SBO) in the parents of a child with an open NTD. ${ }^{16,17}$ The results of these studies suggested SBO, which is considered a type of closed spinal anomaly, occurred more frequently in these parents than in control parents although there were variations in risk according to a variety of covariates such as offspring gender, type of NTD (rostral or caudal) in the offspring, and complexity of SBO in the parent, all serving to highlight the complexity of the entire scenario. Although the estimates of SBO incidence in this population varied between these studies, they do suggest some degree of a genetic relationship between open and this particular closed defect.

Our data provide two separate but related lines of evidence consistent with a common genetic etiology for open and closed neural tube defects in some families. First, the recurrence risk to siblings for open and/or closed neural tube defects is not statistically different than the recurrence risk to siblings with open neural tube defects. Secondly, the familial aggregation presented in several families is further evidence suggesting a common basis. It is interesting to note that there were no families exhibiting complete concordance for the type of NTD within our series of families. In fact, there has only been one reported case of a familial recurrence of lipomyelomeningocele $^{18}$; in this report, the prenatal identification of a fetus with lipomyelomeningocele was followed by the prenatal diagnosis of lipomyelomeningocele in a subsequent pregnancy. Although our findings are consistent with a common genetic etiology, other explanations such as a common environmental exposure or chance association cannot be ruled out.

There are several limitations inherent to this study. First, the sample size is relatively small. Although efforts were made to ascertain participants from a variety of sources, the comparatively low incidence of lipomyelomeningocele and the diagnostic difficulties associated with this condition makes obtaining a large sample size difficult. Second, it is possible our recurrence risk calculations may underestimate the true recurrence risk because asymptomatic cases of closed NTD may be undetected.

In conclusion, it appears lipomyelomeningocele may share at least some of the same genetic factors as open NTDs in some families. This finding may lead to the conclusion that folate supplementation may reduce risk for LMM in some findings; however, McNeely and Howes ${ }^{19}$ recently reported no reduction in LMM prevalence after folate supplementation in Nova Scotia. It is possible that that are mechanisms for LMM that differ between families, some of which are responsive to folate and others not.

Previous studies suggest this relationship extends beyond lipomyelomeningocele to include other forms of closed NTDs, such as SBO and split cord malformation. Families such as these with presumptive variable presentation will be invaluable for identifying and characterizing whether or not such pleiotropic effects exist once genes for open NTDs are found. Larger samples will be necessary before these results can be clinically applied in a counseling setting regarding recurrence risks

\section{APPENDIX}

NTD Collaborative Group: Joanna Aben, RN, Children's Rehabilitation Service, Birmingham, Alabama; Arthur Aylsworth, MD, Cynthia Powell, MD, University of North Carolina, Chapel Hill, North Carolina; Joanne Mackey, RN, Gordon Worley, MD, Duke University Medical Center; Timothy Brei, MD, Constance Buran, DNS, RN, Indiana University School of Medicine, Indianapolis, Indiana; Joann Bodurtha, MD, Virginia; Mark S. Dias, MD, Penn State Uni- 
versity College of Medicine and the Penn State Milton S. Hershey Medical Center, Hershey, PA; Philip Mack, MD, Elli Meeropol, MD, Shriner's Hospital, Springfield, Massachusetts; Nicole Lasarsky, MS, Carolinas Medical Center, Charlotte, NC; W. Jerry Oakes, MD, University of Alabama, Birmingham, Alabama; Marion Walker, MD, Paula Peterson, RN, University of Utah, Salt Lake City, Utah; Bermans Iskandar, MD, University of Wisconsin Hospitals, Madison, Wisconsin.

\section{ACKNOWLEDGMENTS}

The authors gratefully acknowledge the participation of the families. In addition, the authors acknowledge the support of NS39818, NS26630, and HD39083.

\section{References}

1. Bruce DA, Schut L. Spinal lipomas in infancy and childhood. Childs Brain 1979;5: 192-203.

2. Kanev PM, Bierbrauer KS. Reflections on the natural history of lipomyelomeningocele. Pediatr Neurosurg 1995;22:137-140.

3. Oakes. Management of Spinal Cord Lipomas and Lipomyelomeningoceles. Neurosurgery update II: Vascular, spinal, pediatric and functional neurosurgery. New York City: 1991;345-352.

4. Sutton LN. Lipomyelomeningocele. Neurosurg Clin N Am 1995;6:325-338.

5. Seller MJ. Sex, neural tube defects, and multisite closure of the human neural tube. Am J Med Genet 1995;58:332-336.
6. Main DM, Mennuti MT. Neural tube defects: issues in prenatal diagnosis and counselling. Obstetrics and Gynecology 1986;67:1-16.

7. Hume RF, Drugan A, Reichler A et al. Aneuploidy among prenatally detected neural tube defects. Am J Med Genet 1996;61:171-173.

8. Kennedy D, Chitayat D, Winsor EJT, Silver M, Toi A. Prenatally diagnosed neural tube defects: Ultrasound, chromosome, and autopsy or postnatal findings in 212 cases. Am J Med Genet 1998;77:317-321.

9. Juriloff DM, Harris MJ. Mouse models for neural tube closure defects. Hum Mol Genet 2000;9:993-1000.

10. Copp AJ, Greene ND, Murdoch JN. The genetic basis of mammalian neurulation. Nat Rev Genet 2003;4:784-793.

11. Elwood JM, Little J, Elwood JH. Epidemiology and control of neural tube defects. Oxford, UK: Oxford University Press; 1992.

12. George TM, Wolpert CM, Worley G, Mackey JF, Fuchs HE, Speer MC. Variable presen tation of Neural Tube Defects in Three Families. Am J Hum Genet 1996;59:A93.

13. Carter CO, Evans KA, Till K. Spinal dysraphism: Genetic relation to neural tube malformations. J Med Genet 1976;13:343-350.

14. Carter CO, David PA, Laurence KM. A family study of major central nervous system malformations in South Wales. J Med Genet 1968;5:81-106.

15. Melvin EC, Scott WK, Speer MC, Wolpert C, Pericak-Vance MA. Development of a standardized family history questionnaire for research purposes. J Genet Counsel 1998;7:475.

16. Gardner RJM, Alexander C, Veale AMO. Spina bifida occulta in the parents of offspring with neural tube defects. J Genet Hum 1974;22:389-395.

17. Lorber J, Levick K. Spina bifida cystica: incidence of spina bifida occulta in parents and in controls. Archives of Disease in Childhood 1967;42:171-173.

18. Seeds JW, Powers SK. Early prenatal diagnosis of familial lipomyelomeningocele. Obstet Gynecol 1988;72:469-471.

19. McNeely PD, Howes WD. Ineffectiveness of dietary folic acid supplementation on the incidence of lipomyelomeningocele pathogenetic implications. J Neurosurg 2004;100:98-100. 